\title{
Nanoplasmonic Colloidal Suspensions for the Enhancement of the Single Walled Carbon Nanotubes Luminescent Emission
}

\author{
Mareen Glaeske $^{1}$ and Antonio Setaro ${ }^{1}(\bowtie)$ \\ ${ }^{1}$ Department of Physics, Freie Universität Berlin, Arnimallee 14, 14195 Berlin (Germany)
}

\begin{abstract}
Aiming to enhance the luminescence yield of carbon nanotubes, we introduce a new class of hybrid nanoplasmonic colloidal systems ( $\pi$-hybrids). Nanotubes dispersed in gold nanorod colloidal suspensions yield hybrid structures exhibiting enhanced luminescence up to a factor of 20 . The novelty of the proposed enhancement mechanism relies on including metal proximity effects in addition to its localized surface plasmons. This simple, robust and flexible technique enhances the luminescence of nanotubes with chiralities whose enhancement has never reported before, for example the $(8,4)$ tube.
\end{abstract}

\section{KEYWORDS}

Carbon Nanotubes, Luminescence Enhancement, Gold Nanorods, Nanoplasmonic Colloids, Hybrid Structures

\section{Introduction}

In the world at nano scale, carbon nanotubes (CNTs) stand out for their exceptional mechanical, optical, and electronic properties [1]. Single walled carbon nanotubes (SWNTs) can be seen as a graphene sheet cut and rolled up along a certain direction, described by the so-called chiral vector $\mathbf{c}=\left\{\mathrm{n}_{1}, \mathrm{n}_{2}\right\}$. The boundary conditions resulting from this process introduce quantization of the tube's allowed electronic states. As a consequence, there are both nanotubes with metallic character and nanotubes with a band-gap. In semiconducting nanotubes, luminescence can be observed. The photon energies involved in the process uniquely identify a tube with a given chirality [2],[3]. The photo-generated electrons and holes form strongly bounds excitons with binding energies from few tens of meV up to $1 \mathrm{eV}$, which dominate the optical spectra of SWNTs [4],[5]. The photoluminescence (PL) yield of SWNTs is very low typically around 3\% for single suspended nanotubes, or $0.05 \%$ for nanotube solutions [6]-[8]. The highest yield reported so far is $20 \%$ for CNTs dispersed with special oxygen-excluding surfactants [9]. Several routes have been pursued to overcome this limit and enhance the luminescence of the tubes, for example through energy transfer between dye molecules and SWNTs [10], by including tubes into optical cavities made with metallic [11] or dielectric [12] mirrors, and by exploiting photonic crystals [13]. The

Address correspondence to Antonio Setaro, setaro@physik.fu-berlin.de. 
luminescence enhancement achieved in all these experiments is driven by the same physical two mechanisms, namely either stronger pumping of the excited state or speeding up the radiative de-excitation rate. The metal-induced PL enhancement seems to be particularly promising, because, engineering the experimental parameters, both mechanisms contribute to the luminescence enhancement. Metal-induced enhancement was shown to enhance PL signals for molecular fluorophores. It is based on the fact that the proximity of the metal surface will cause additional de-excitation rate [14]. Under the proper conditions [15], this additional de-excitation rate due to the metal presence will cause a shortening of the decay times as well as an enhancement of the PL quantum yield. Additionally, the quantum yield can be further affected by exploiting structured metal surfaces, where the electric fields get strongly enhanced through bright localized surface plasmons (LSPs). The surface morphology, the plasmon wavelength $\lambda \pi$ and the electric field enhancement are the key parameters in such systems. Adapting the expression in Ref. [16], the emission quantum yield, given by the PL enhancement factor, can be written as:

$$
Q_{m, \pi}=\operatorname{Im} / I_{0}=\eta(\lambda \pi, d) Q m(d),
$$

where $Q_{m}(d)=\left(\gamma_{R}+\gamma_{N R}\right) /\left(\gamma_{R}+\gamma_{N R}+\gamma_{m}(d)\right)$ accounts for the proximity effects and $\gamma s$ are the decay rates (respectively, $\gamma_{\mathrm{R}}$ is radiative, $\gamma_{\mathrm{NR}}$ non-radiative, and $\gamma_{\mathrm{m}}$ due to the exciton transfer from the molecule to the metal surface). $\eta\left(\lambda_{\pi}, d\right)=\left|E(\lambda \pi, d) / E_{0}\right|^{2}$ is the electric field enhancement observed in case the wavelength of the exciting laser matches the plasmonic features of the metallic system. Thus the nanostructuring of the metal surfaces will give an extra contribution to the metal-enhancement of the optical properties of a fluorophore. Please note that LSPs require a certain tube-metal separation (cfr. Ref. [16]) in order to induce PL enhancement. For this reason we will refer in the rest of the paper to plasmonic enhancement as to the contribution to the LSPs while we will refer as proximity effects for the other mechanisms. It is worth noting the neat effect of the two terms in Eq. (1), as they will be easily distinguished in a photoluminescence excitation (PLE) chart: While $Q_{m}$ depends upon both metal and fluorophore, $\eta$ depends only upon the metal. The intensity-enhanced localized surface plasmons, which lead to a stronger pumping of the fluorophore, possess a specific wavelength $\lambda_{\pi}$. Hence only pumping the system at this specific wavelength the enhanced pumping mechanism occurs: The effect will thus appear along the horizontal direction in a PLE chart (i.e. at a fixed excitation wavelength). The term $Q_{m}$ containing the proximity effects is observable everywhere in the chart, not being limited to certain excitation wavelengths.

Various nanostructured metal systems have been synthesized. An inspiring review on this topic can be found in Ref [17]. The value of the field enhancement and the coupling with the fluorophores depend upon the morphology of the nanostructured material; the details remain an open theoretical and computational challenge.

The metal-enhanced fluorescence approach was exploited to enhance the luminescence of more elaborate structures than simple molecular fluorophores. A three-fold enhancement of the $(7,5)$ SWNT PL emission intensity was observed on a sputtered $\mathrm{Au}$ surface [16], whereas a ten-fold enhancement of tubes with $E_{22}$ transition energies around $650 \mathrm{~nm}[(7,5),(7,6)$, and $(10,3)]$, was reported for a highly porous surface directly grown from $\mathrm{Au}$ nanoparticles [18]. In both studies, the PL was enhanced for nanotubes of specific chiralities, whose electronic properties matched the frequency window of the surface plasmons. These chiralities all lie along a single excitation line, i.e. $650 \mathrm{~nm}$, in a PLE chart. The physical mechanism behind the enhancement is rooted in the $\eta\left(\lambda_{\pi}, d\right)$ term in Eq. (1).

The key idea of the present work lies in the growth of gold nanoparticles with tailored features.

Our approach is doubly advantageous: By rationally synthesizing the gold nanostructure with specific plasmonic features, we address carbon nanotubes of targeted chiralities, accessing also those tubes outside the resonance window of the previous experimental reports. Moreover, by tuning the aspect ratio and shape of the nanoparticle, we realize 


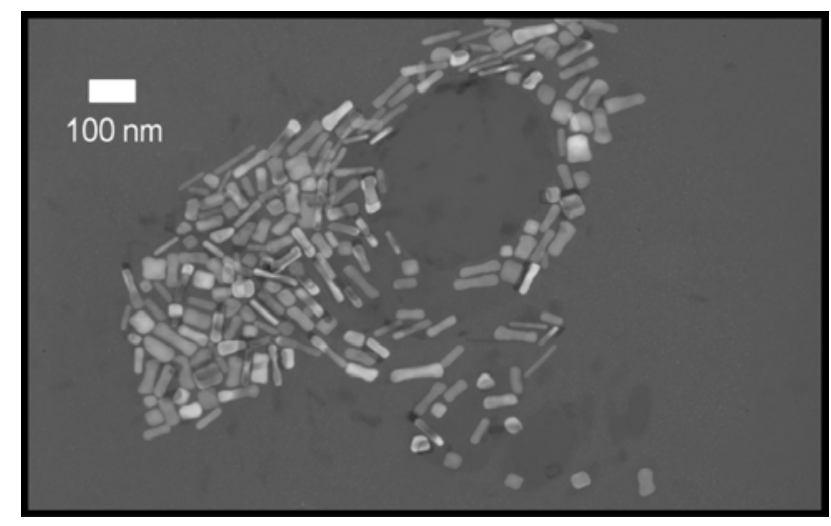

Figure 1 TEM picture of the AuNR synthesized for this work. The rod particles are 100nm long and 20nm width on the average.

structures with improved electric field enhancement. In this way we take advantage of both terms in Eq. (1) for achieving optimal enhancement. Another peculiarity of our approach lies in the fact that, instead of casting the gold nanostructures on a surface, we keep them in aqueous solution, realizing a nanoplasmonic colloidal suspension.

Nanoplasmonic colloids were successfully exploited to enhance the fluorescence of several systems, in particular in systems of biological interest, where working in a water suspension is fundamental [19]. CNTs have been profitably included in biological systems [20]-[22]. Including luminescence-enhanced SWNTs in nanoplasmonic colloidal dispersions and creating hybrid plasmonic ( $\pi$-hybrids) colloids would help implementing suspensions with bright markers emitting in the near-infrared window of biological tissues. Please note that the $\pi$ of our $\pi$-hybrids has been chosen to highlight their plasmonic character and does not refer to the nature of the chemical bond holding the constituents together. A last advantage of the proposed system is its robustness and flexibility: While other enhancement methods do work in special configurations realized for the fundamental investigations but cannot be integrated in real systems without loss of the enhancement, the colloidal suspensions can be profitably integrated without loss, for example, in biological systems [19].

\section{Experiments and discussion}

We worked with gold nanorods (AuNR) for sake of simplicity of preparation and characterization. For details on the synthesis and characterization of the colloidal suspension made by suspending AuNR with $\mathrm{CTAB}$ in water, please see the Supporting Information file. We will refer to the AuNR suspension for the rest of this paper as the mother solution. A TEM picture of the AuNR synthesized for this work can be found in Fig. 1. The resulting AuNRs had an average length of $100 \mathrm{~nm}$ and average width of $20 \mathrm{~nm}$ (see Fig. 1) and the resonance wavelength of the Longitudinal (LP) and Transverse Plasmons (TP) were, respectively, $740 \mathrm{~nm}$ and $520 \mathrm{~nm}$, as it can be seen in Fig. 2(a). The nanotube solubilization was performed following the stirring method in CTAB reported in Ref. [23] and an adapted version of the stirring method was exploited in order to promote the $\pi$-hybrid formation. Details on the experimental procedure can be found in the Supporting Information. It is worth noting that this is the only method promoting the hybrid formation; sonication, for example, does not yield any hybrids. Basically, our hybridization process can be seen as an (a)

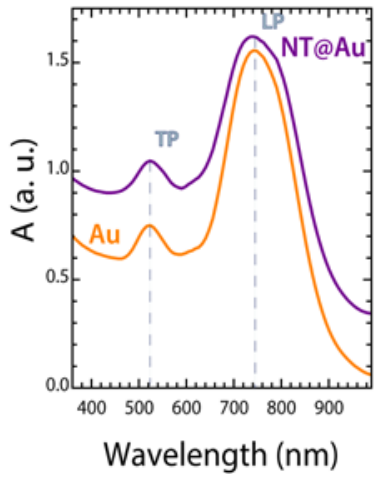

(b)

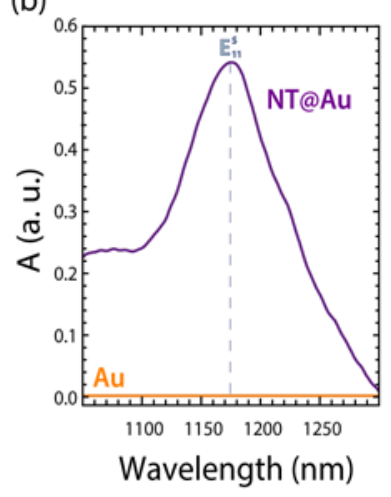

Figure 2 (a) Absorption spectra of the AuNR suspension (orange curve labeled as $\mathrm{Au}$ ) and of the SWNT-AuNRs complexes (purple curve labeled as NT@Au). The two absorption bands called TP and LP correspond to the transversal and longitudinal plasmon frequencies. (b) IR Absorption spectra of the AuNR suspension (orange curve labeled as $\mathrm{Au}$ ) and of the SWNT@Au complexes (purple curve labeled as NT@Au). In this region, the gold does not absorb and only the $\mathrm{E}_{11}{ }^{\mathrm{S}}$ nanotube absorption band can be observed. 
adapted version of the micelle swelling technique [24]: In a water suspension of micelle-stabilized nano-objects (in our case, the CTAB-stabilized AuNRs) we add some additional, non-water soluble objects (in our case, the SWNTs). As the tubes are not soluble in water, they can either (a) precipitate or (b) be encapsulated within the gold-containing micelles. The micelle filling process has to be performed by following the gentle stirring procedure, otherwise the AuNR-containing micelle get broken, the equilibrium and composition of the object suspended in the samples get altered and Au precipitation can be observed. This is the reason why the sonication method cannot lead to any hybrid formation in our case. This process of encapsulation of the tubes within the micelles can go on up to a certain limit of the micelle loading. There is thus a certain amount of tubes to add to the starting solution beyond which no further encapsulation is possible and the excess of tubes will start precipitating. In the following, we will refer to the $\pi$-hybrid colloidal dispersion as NT@Au. Please note that the visible part of the absorption spectrum of the NT@Au suspension is dominated by the plasmonic features of the gold nanorods (Fig. 2(a)). In the NIR part of the spectrum, on the contrary, the $\mathrm{E}_{11} \mathrm{~s}$ transition bands of the semiconducting SWNTs are the predominant feature (Fig. 2(b)). As no contribution from Au absorption occurs in this range, the $E_{11} \mathrm{~S}$ band can be usefully exploited as indicator of the total amount of tubes encapsulated within the $\pi$-hybrids.

As the metal-fluorophore distance plays an important role in the metal-enhancement of the luminescence, we decided to change the nanotube density in the solution in order to try and affect the tubes-to-rods relative ratio. In Fig. $3(\mathrm{~b})$ the PL intensity of the $(7,5)$ and $(7,6)$ tubes is shown for different amounts of tubes added to the mother solution. In the following, we will refer to the SWNTs stirred in the mother solution as NT@Au. In a typical suspension of SWNTs sonicated in CTAB the intensity of the $(7,5)$ peak is 1.5 times stronger than the $(7,6)$ one. As visible in Fig. $3(b)$, in the NT@Au samples the intensity ratio between the two emission bands is inverted, which is already a first

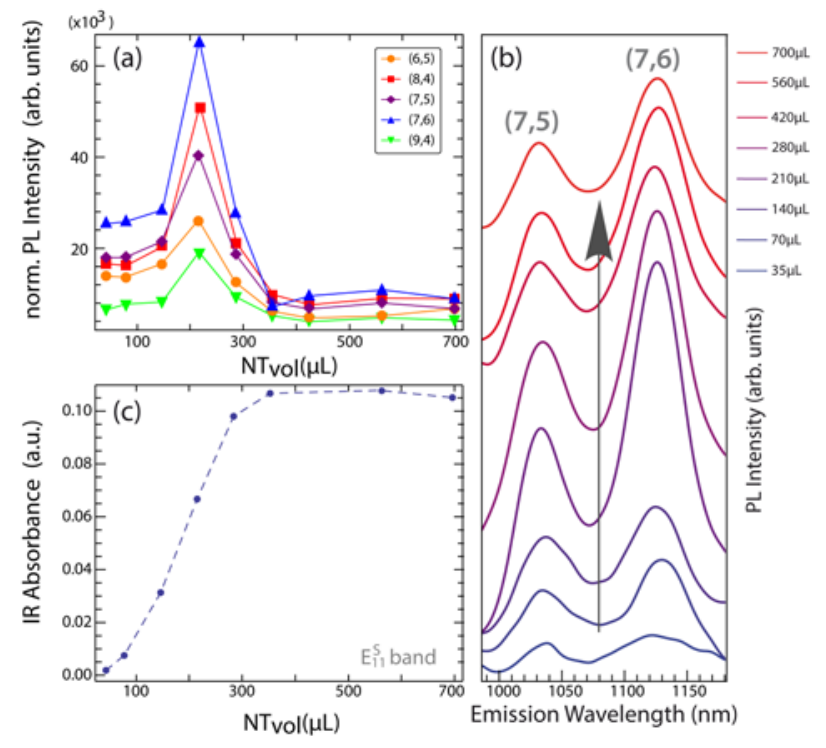

Figure 3 (a) Normalized intensity of the PL emission of tubes with different chiralities for different amount of SWNTs added to the mother solution. The spectra have been normalized by the density of tubes in the final suspensions. (b) PL emission of the $(7,5)$ and $(7,6)$ tubes for different starting SWNTs concentration. The traces are offset for clarity. (c) Intensity of the $E_{11}{ }^{S}$ absorption band at $1175 \mathrm{~nm}$ as an estimation of the amount of tubes present in the final suspensions.

indication that the interaction with the AuNRs is actually affecting the tubes. The inversion of the intensity of the $(7,5)$ and $(7,6)$ tubes is accompanied by a nonmonotonous change of the peak intensities when tuning the nanotube-to-nanorod ratio. When increasing the fraction, PL emission increases up to a certain level before it starts to decrease again. In Fig. 3(a) the intensities of the PL for all the tubes present in solution are reported; the spectra have been normalized by the density of tubes in the final suspensions for estimating of the average emission per tube. The original uncorrected intensities as well as the IR absorption spectra can be found in Fig. S1 of the supporting information. A similar behaviour, with the intensity reaching a maximum and then decreasing, is shared by all chiralities. To rule out that the lowering of the PL intensity results from a loss of material, we compare the PL intensity with the intensity of the $\mathrm{E}_{11} \mathrm{~S}$ absorption band in Fig. 3(c). The absorption can be taken as an indicator of the 


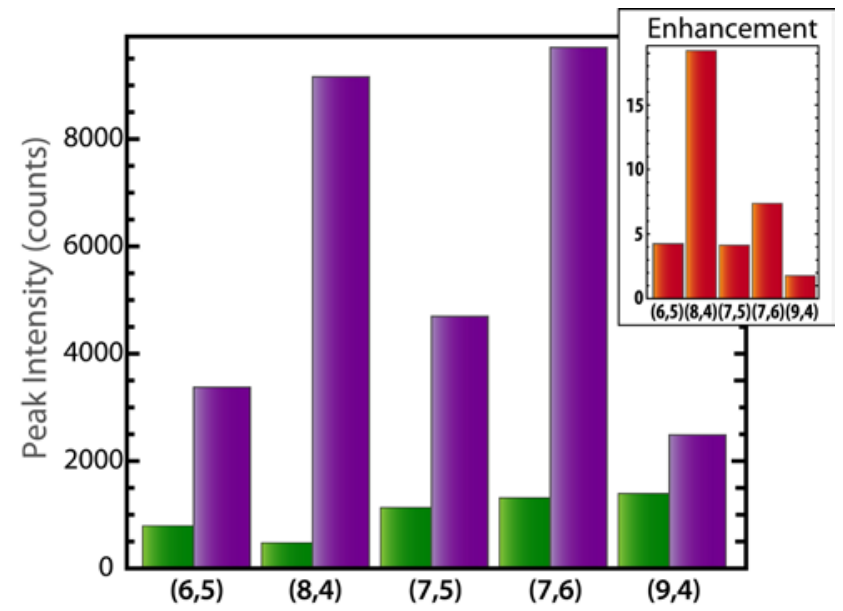

Figure 4 Histogram of the distribution of the PL intensities of the NT@Au (purple) and for the NT@CTAB (green) samples.

The inset reports the histogram of the distribution of the enhancement of NT@Au intensity with respect to the NT@CTAB sample.

amount of the tubes present in solution [23]. Starting from $210 \mu \mathrm{L}$ and adding bigger amount of tubes, we observe that the overall luminescence of the tubes decreases (cfr. Fig. 3(a)) while the amount of tubes in suspension keeps increasing. We assume that in this region the number of SWNTs within the micelles starts becoming high enough to make tube-tube interaction and related quenching phenomena relevant. Starting from amount of tubes bigger than $400 \mu \mathrm{L}$, we assume that we reach the maximum load of the micelles; this is supported by the observation of precipitate formation in our samples, given by the excess of tubes. This accounts also for the observed saturated trend of the absorption spectra (Fig. 3(c)): The density of tubes in the final suspension remains constant, even by starting with higher amount of tubes.

So far we have only reported on the behaviour of the luminescence of the NT@Au; in order to estimate the enhancement of the PL, we compared our best NT@Au samples (the one showing the highest PL intensity, corresponding to $210 \mu \mathrm{L}$ NTvoL) with a sample prepared by following exactly the same procedure but using a solution with only CTAB (without any AuNR) instead of the mother solution. We will refer to such a sample in the rest of the paper as NT@CTAB. Fig. 4 shows the histogram distribution of the intensities of the PL emissions for the different tubes present in our samples. The distribution of the intensities between the various chiralities is altered after the interaction with the AuNRs. The most striking effect, however, is the strong enhancement of the PL emission when the tubes interact with the gold nanoparticles. In the inset of Fig. 4 the histogram distribution of the ratio between the intensities of NT@Au and NT@CTAB is reported. We enhance the PL intensity of the tube chirality $(8,4)$ by a factor of 20 . The $(7,6)$ tube luminescence gets enhanced by a factor of eight, which is a value comparable with the one reported for NT deposited on a porous Au surface [18]. The correlation between enhancement, tube chirality and metallic particle plasmonic features will be object of future investigations. This experiment is a proof of concept for the PL enhancement in $\pi$-hybrids colloidal suspensions. The authors believe that there is still a large margin of improvement by optimizing the amount of AuNR in the mother solution, the morphology of the nanoparticles, as well as the choice of metal.

In order to further confirm the efficiency of the proposed PL enhancing system, we compared our NT@Au sample with SWNTs in a CTAB suspension prepared following the standard preparation (i.e. sonication and centrifugation). For the description of the standard preparation procedure of such a suspension, please refer to any of our past works [25],[26]. We will refer in the text and in the figures to such a sample just as CTAB. The comparison between the NT@Au and the CTAB sample in Fig. 5 highlights the benefits of our systems: By moving along the $\alpha$ excitation lines of the PLE charts -cfr. Fig. 5(c)- one can observe a very pronounced enhancement of the fluorescent emission of the $(7,6)$ and the $(8,4)$ chiralities. Surprisingly, no enhancement for the $(9,4)$ tube can be observed. Similarly, by moving along the $\beta$ emission lines (Fig. 5(d)), the intensity of the $(7,5)$ tube is approximately the same in both samples while the $(7,6)$ gets enhanced. This behavior highlights the originality of our system: The enhancement occurs in several regions of the PLE chart, revealing that the $\pi$-hybrids take advantage of proximity effects (the $Q_{m}$ term in Eq. (1)), unlike previous works [16],[18] that 
(a)
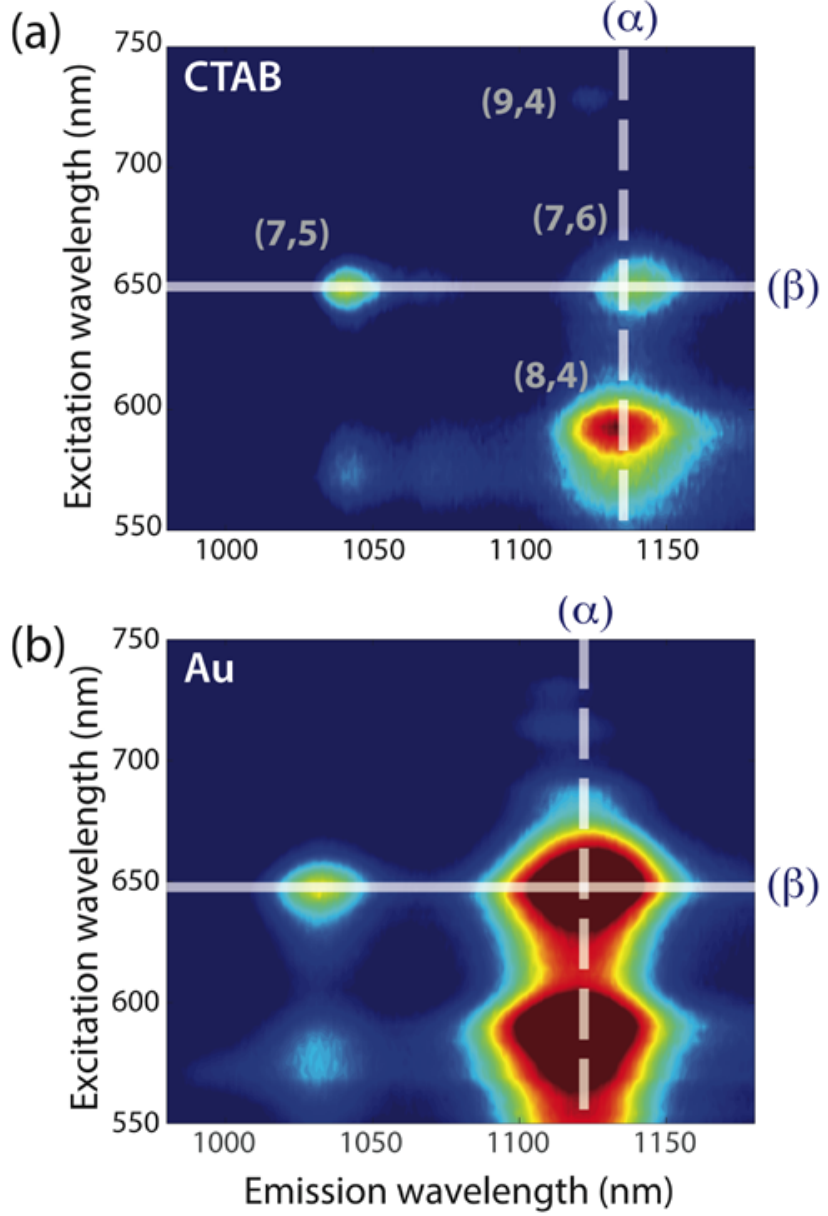
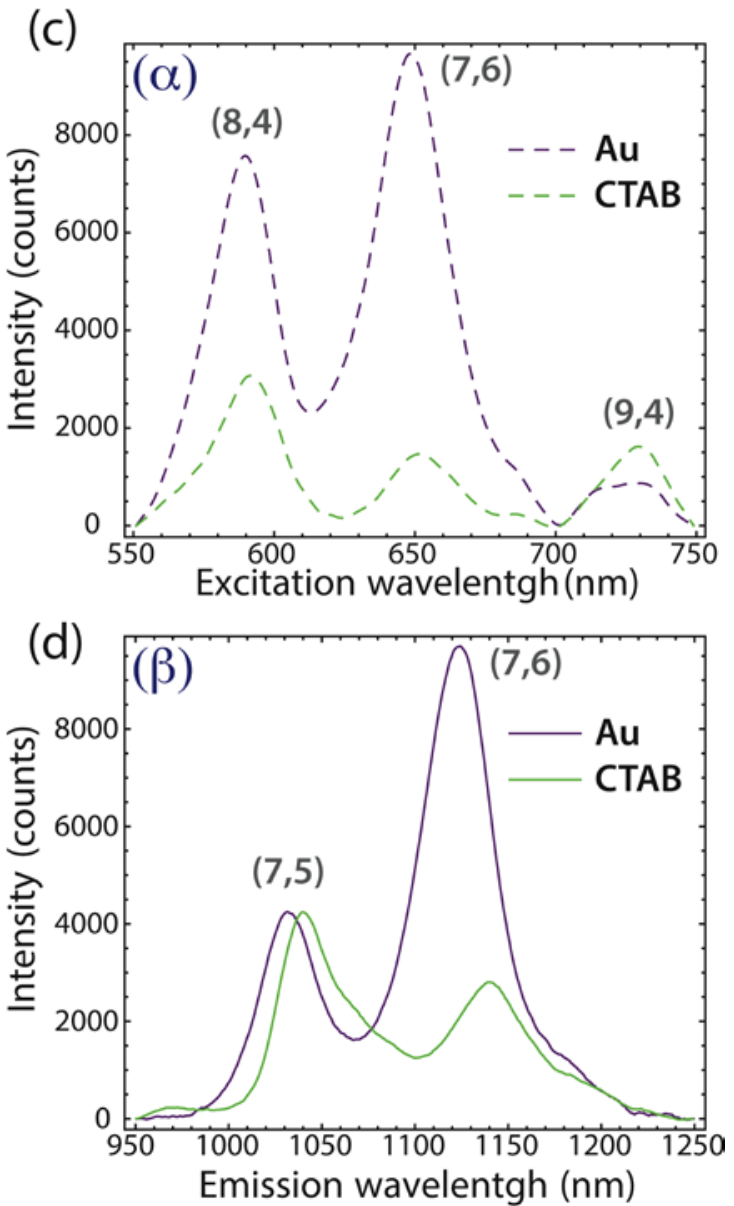

Figure 5 (a) PLE chart of the CTAB sonicated sample. (b) PLE chart of the NT@Au sample. The dotted lines denoted with an ( $\alpha$ ) represent a single excitation line taken in at the maximum of the $(7,6),(8,4)$ emission intensities, while the continuous lines denoted with a $(\beta)$ are single emission lines taken at the excitation maximum of the $(7,5),(7,6)$ tubes. Please note that the emission wavelength of the NT@Au sample differ from the one of the CTAB sample, indicating an interaction with the AuNRs. (c) Comparison of the single excitation lines of the NT@Au sample with the CTAB sample. (d) Comparison of the single emission lines of the NT@Au sample with the CTAB sample.

only resorted to localized surface plasmons (the $\eta$ term in Eq. (1)) and was limited to the $650 \mathrm{~nm}$ excitation line. It is worth pointing out that the spectral position of the PLE peaks in addition to their relative intensities are altered by the presence of AuNRs. Surprisingly, the emission wavelength is strongly affected while the excitation is not. This rules out the solvatochromic shift [27] (which would affect in a similar fashion both excitation and emission wavelengths), or electric dipole-nanotube interaction [28]-[31]. Indeed, this behaviour has been observed and modelled for an analogous system constituted by a low-quantum yield nanostructured semiconductor (CdTe nanowires) interacting with gold nanoparticles [32]. At an optimal semiconductor-metal distance, the sample exhibited the same phenomenology as our samples. This has been proven [32] to be consequence of the excitons-plasmon interaction in the hybrid superstructure: At an optimal metal-semiconductor distance, a significant shortening of the excitonic lifetimes occurs with 
consequent blue shift of the emitted spectra. The presence of the blue shift in the spectra emitted by our $\pi$-hybrids can be depicted in the framework of the results of Ref. [32] as consequence of the nanotube-exciton metal-plasmon coupling. Please note that no overlap between gold plasmonic features and nanowires energy bands are present in the experiment of Ref. [32]; we assume that this is the reason why no enhancement of the luminescence has been observed there.

In order to investigate the relation of the emission enhancement and blue shifts vs. interaction strengths between the constituents of the $\pi$-hybrids, several sonication steps were applied to the stirred samples NT@Au and NT@CTAB. Sonication is a high-energetic treatment that does not lead to any hybrid formation and any metal-induced PL enhancement (see Supporting Information) and is thus expected to promote further isolation of tubes from small bundles and to possibly break some $\pi$-hybrids into their original constituents. For this reason, sonication can be used as a tool for testing the strength of the interaction yielding the $\pi$-hybrids. The emission bands of the $(7,5)$ and $(7,6)$ chiralities have been monitored during the process and are reported in Fig. 6. The solutions were exposed to short sonication periods of, respectively, 2,4 , and $14 \mathrm{~min}$. Noticeably, this simple experiment reveals different trends for the $(7,5)$ and the $(7,6)$ tubes: The emitted intensity of the $(7,5)$ tube behaves in a similar manner in both samples with and without gold, increasing with increasing sonication times. The intensity of the $(7,6)$ tube, on the contrary, behaves differently in the Au@NT and CTAB samples: In the gold solution its intensity remains the same, unaffected by the sonication steps (cfr. Fig. 6(a)). Also the spectral position of the bands reveals different trends: after just the first sonication step, the spectral position of the $(7,5)$ tube in the NT@Au suspension reaches the position of the free $(7,5)$ tube and no blue shift can be observed through the other sonication steps (cfr. Fig. $6(\mathrm{~b})$ and $(\mathrm{c}))$. For the $(7,6)$ tube, on the contrary, the spectral position of the sample still evolves under the different sonication steps (cfr. Fig. 6(b))
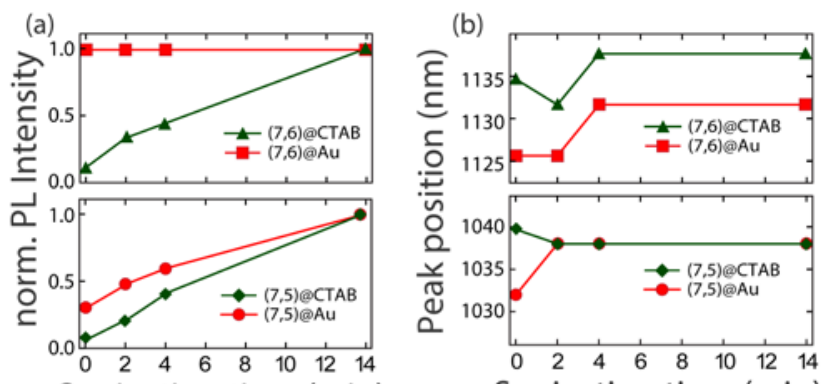

Sonication time ( $\mathrm{min})$

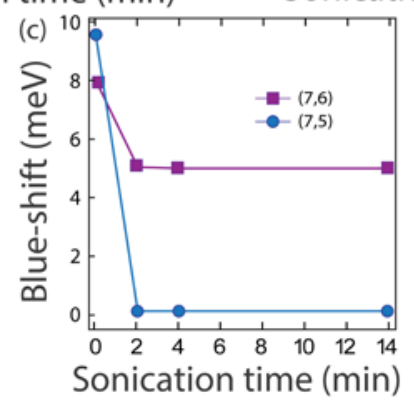

Figure 6 (a) Intensity of the PL emission bands of the $(7,5)$ and $(7,6)$ tubes (normalized by setting to 1 the intensity after the last sonication step) of the NT@Au and NT@CTAB samples after different sonication steps (0, 2, 4, and 14min, respectively), (b) Spectral position of the PL emission bands of the $(7,5)$ and $(7,6)$ tubes of the NT@Au and NT@CTAB samples after the different sonication steps, and (c) Au-induced blue shifts of the emission bands of the $(7,5)$ and $(7,6)$ tubes after the different sonication steps.

but the blue shift between the emission of the tube in the NT@Au and the NT@CTAB solutions persists and is not destroyed by the sonication treatment (cfr. Fig. 6(c)). This different response suggests that the $(7,5) @$ Au complex is weakly bound and can easily get broken while the $(7,6) @ A u$-hybrid is strongly bound, does not get set apart and remains unperturbed even after sonication. It is worth pointing out that binding strength and PL enhancement are strictly related in our samples.

In order to draw any general conclusion, anyway, analysis of samples with a broader set of chiralities needs to be carried out. The systematic correlation between PL enhancement, blue shifts, stability of the complexes and the selective chemistry behind these phenomena will be object of future investigations. 


\section{Conclusions}

In conclusion, by exploiting a nanoplasmonic colloidal suspension of gold nanorods, we have implemented $\pi$-hybrid (gold nanorod-carbon nanotubes hybrid structures) colloidal suspensions which exhibit a 20-fold enhanced photoemission with respect to isolated single walled carbon nanotubes. The physical mechanisms behind the achieved enhancement can be ascribed to more complex phenomena than the previously reported mechanisms: Not only surface localized electric fields but also proximity effects do contribute to the overall enhancement effect. Altering the morphology of the nanoplasmonic particle may result in an even higher contribution to the luminescence enhancement due to the stronger localized electric fields [33]. Working in suspension offers the advantage of easing experimental access to the fundamental optical properties of the $\pi$-hybrids. Moreover, this approach yields strongly IR-luminescent suspensions that can profitably be integrated in biological systems. The potential of this technique lies in its simplicity, robustness, and flexibility, as a huge variety of metal nanostructured particles can be easily synthesized with plasmonic and morphological features tailored to match nanotubes with targeted chiralities. We have proven this principle in the enhancement of the luminescence of the $(8,4)$ tube, whose luminescence enhancement has never been reported before in literature.

\section{Acknowledgements}

The authors gratefully thank the FU Focus Area NanoScale for financial support; parts of the work were supported by the DFG under the SfB 658 .

The authors thank P. Bluemmel, F. Ernst, and S. Heeg for useful discussions and S. Reich for a critical reading of the manuscript.

\section{Electronic Supplementary Material:}

Supplementary material describing the experimental procedure towards the gold nanorod synthesis, the hybridization route as well as the characterization setup is available in the online version of this article at www.

\section{References}

[1] Reich, S; Thomsen, C.; Maultzsch J., Carbon nanotubes: basic concepts and physical properties; Wiley-VCH; 2004.

[2] O’Connell, M. J.; Bachilo, S.M.; Huffman, C.B.; Rialon, K.L.; Boul, P.J.; Noon, W.H. Band Gap Fluorescence from Individual Single-Walled Carbon Nanotubes, Science 2002, 297, 593.

[3] Bachilo, S.M.; Strano, M.S.; Kittrell, C.; Hauge, R.H.; Smalley, R. E.; Weisman, R.B. Structure-Assigned Optical Spectra of Single-Walled Carbon Nanotubes, Science 2002, 298, 2361.

[4] Maultzsch, J.; Pomraenke, R.; Reich, S.; Chang, E.; Prezzi, D.; Ruini, A.; Molinari, E.; Strano, M.S.; Thomsen, C.; Lienau, C. Exciton binding energies in carbon nanotubes from two-photon photoluminescence, Phys. Rev. B 2005, 72, 241402.

[5] Wang, F.; Dukovic, G.; Brus, L.E.; Heinz, T.F. The Optical Resonances in Carbon Nanotubes Arise from Excitons, Science 2005, 308, 838.

[6] Ernst, F.; Heek, T.; Setaro, A.; Haag, R.; Reich, S. Energy Transfer in Nanotube-Perylene Complexes, Adv. Funct. Mat. 2012, 22, 3921.

[7] Carlson, L.J.; Maccagnano, S.E.; Zheng, M.; Silcox, J.; Krauss, T.D. Fluorescence Efficiency of Individual Carbon Nanotubes, Nano Letters 2007, 7, 3698.

[8] Jones, M.; Engtrakul, C.; Metzger, W.K.; Ellingson, R.J.; Nozik, A.J.; Heben, M.J.; Rumbles, G. Analysis of photoluminescence from solubilized single-walled carbon nanotubes, Phys. Rev. B 2005, 71, 115426.

[9] Ju, S., Kopcha, W., Papadimitrakopoulos, F., Brightly fluorescent single-walled carbon nanotubes via an oxygen-excluding surfactant organization. Science, 2009 323, 1319.

[10] Ahmad, A.; Kern, K.; Balasubramanian, K. Selective enhancement of carbon nanotube photoluminescence by resonant energy transfer, ChemPhysChem 2009, 10, 905. 
[11] Xia, F.; Steiner, M.; Lin, Y.M.; Avouris, P. A microcavity-controlled, current-driven, on-chip nanotube emitter at infrared wavelengths, Nat Nano 2008, 3, 609.

[12] Gaufrès, E.; Izard, N.; Roux, X.L.; Kazaoui, S.; Marris-Morini, D.; Cassan, E.; Vivien, L. Optical microcavity with semiconducting single-wall carbon nanotubes, Opt. Express 2010, 18, 5740.

[13] Watahiki, R.; Shimada, T.; Zhao, P.; Chiashi, S.; Iwamoto, S.; Arakawa, Y.; Maruyama, S.; Kato, Y.K. Enhancement of carbon nanotube photoluminescence by photonic crystal nanocavities, Applied Physics Letters 2012, 101, 141124.

[14] Lakowicz, J.R.; Shen, Y.; D’Auria, S.; Malicka, J.; Fang, J.; Gryczynski, Z.; Gryczynski, I. Radiative Decay Engineering - 2. Effects of Silver Island Films on Fluorescence Intensity, Lifetimes, and Resonance Energy Transfer, Analytical Biochemistry 2002, 301, 261.

[15] Ming, T.; Chen, H.; Jiang, R.; Li, Q.; Wang, J. Plasmon-Controlled Fluorescence: Beyond the Intensity Enhancement, The Journal of Physical Chemistry Letters 2012, 3, 191.

[16] Sakashita, T.; Miyauchi, Y.; Matsuda, K.; Kanemitsu, Y. Plasmon-assisted photoluminescence enhancement of single-walled carbon nanotubes on metal surfaces, Applied Physics Letters 2010, 97, 063110.

[17] Halas, N.J. Plasmonics: An Emerging Field Fostered by Nano Letters, Nano Letters 2010, 10, 3816.

[18] Hong, G.; Tabakman, S.M.; Welsher, K.; Wang, H.; Wang, X.; Dai, H.; Matsuda, K.; Kanemitsu, Y.; Irie, K.; Saiki, T.; Someya, T.; Miyauchi, Y.; Maruyama, S. Metal-enhanced fluorescence of carbon nanotubes, JACS 2010, 132, 15920.

[19] Deng, W.; Goldys, E.M. Plasmonic Approach to Enhanced Fluorescence for Applications in Biotechnology and the Life Sciences, Langmuir 2012, 28, 10152.

[20] Cherukuri, P.; Bachilo, S.M.; Litovsky, S.H.; Weisman, R.B. Near-Infrared Fluorescence Microscopy of Single-Walled Carbon Nanotubes in Phagocytic Cells, JACS 2004, 126, 15638.

[21] Leeuw, T.K.; Reith, R.M.; Simonette, R.A.; Harden, M.E.; Cherukuri, P.; Tsyboulski, D.A.; Beckingham, K.M.;
Weisman, R.B. Single-Walled Carbon Nanotubes in the Intact Organism: Near-IR Imaging and Biocompatibility Studies in Drosophila, Nano Letters 2007, 7, 2650.

[22] Welsher, K.; Liu, Z.; Sherlock, S.P.; Robinson, J.T.; Chen, Z.; Daranciang, D.; Dai, H. A route to brightly fluorescent carbon nanotubes for near-infrared imaging in mice, Nat Nano 2009, 4, 773.

[23] Wenseleers, W.; Vlasov, I; Goovaerts, E.; Obraztsova, E.; Lobach, A.; Bouwen, A. Efficient Isolation and Solubilization of Pristine Single-Walled Nanotubes in Bile Salt Micelles, Advanced Functional Materials 2004, 14, 1105 .

[24] Wang, R.K.; Chen, W.C.; Campos, D.K.; Ziegler, K.J. Swelling the Micelle Core Surrounding Single-Walled Carbon Nanotubes with Water-Immiscible Organic Solvents. Journ. Am. Chem. Soc. 2008, 130, 16330-16337.

[25] Setaro, A.; Popeney, C.S.; Trappmann, B.; Datsyuk, V.; Haag, R.; Reich, S. Polyglycerol-derived amphiphiles for single walled carbon nanotube suspension, Chemical Physics Letters 2010, 493, 147.

[26] Popeney, C.S.; Setaro, A.; Mutihac, R.C.; Bluemmel, P.; Trappmann, B.; Vonneman, J.; Reich, S.; Haag, R. Polyglycerol-Derived Amphiphiles for the Solubilization of Single-Walled Carbon Nanotubes in Water: A Structure-Property Study, ChemPhysChem 2012, 13, 203.

[27] Choi, J.H.; Strano, M.S. Solvatochromism in single-walled carbon nanotubes, Applied Physics Letters 2007, 90, 223114.

[28] Malic, E.; Weber, C.; Richter, M.; Atalla, V.; Klamroth, T; Saalfrank, P.; Reich, S.; Knorr, A. Microscopic Model of the Optical Absorption of Carbon Nanotubes Functionalized with Molecular Spiropyran Photoswitches, Phys. Rev. Lett. 2011, 106, 097401.

[29] Setaro, A.; Bluemmel, P.; Maity, C.; Hecht, S.; Reich, S. Non-Covalent Functionalization of Individual Nanotubes with Spiropyran-Based Molecular Switches, Advanced Functional Materials 2012, 22, 2425.

[30] Bluemmel, P.; Setaro, A.; Maity, C.; Hecht, S.; Reich S. Tuning the interaction between carbon nanotubes and dipole switches: the influence of the change of the 
nanotube-spiropyran distance, Journal of Physics: Condensed Matter 2012, 24, 394005.

[31] Cambré, S.; Santos, S.M.; Wenseleers, W.; Nugraha, A.R.T.; Saito, R.; Cognet, L.; Lounis, B. Luminescence Properties of Individual Empty and Water-Filled Single-Walled Carbon Nanotubes, ACS Nano 2012, 6, 2649.
[32] Lee, J.; Hernandez, P.; Lee, J.; Govorov, A.O.; Kotov, N.A. Exciton-plasmon interactions in molecular spring assemblies of nanowires and wavelength-based protein detection, Nat Mater 2007, 6, 291.

[33] Kou, X.; Ni, W.; Tsung, C.K.; Chan, K.; Lin, H.Q.; Stucky, G.; Wang, J. Growth of Gold Bipyramids with Improved Yield and Their Curvature-Directed Oxidation, Small 2007, 3, 2103. 


\title{
Electronic Supplementary Material
}

\section{Nanoplasmonic Colloidal Suspensions for the Enhancement of the Single Walled Carbon Nanotubes Luminescent Emission}

\author{
Mareen Glaeske ${ }^{1}$ and Antonio Setaro ${ }^{1}(\bowtie)$ \\ ${ }^{1}$ Department of Physics, Freie Universität Berlin, Arnimallee 14, 14195 Berlin (Germany) \\ Supporting information
}

\section{SETUP AND EXPERIMENTAL DETAILS.}

CoMoCat ${ }^{\circledR}$ SWNTs produced by SWeNT® were used for this experiment.

Photoluminescence and photoluminescence excitation (PLE) experiments were performed with a Fluorolog-3 Spectrofluometer (Horiba Jobin Yvon). A Xenon lamp with emission range 240-1000 nm provided the optical excitation. The light was focused onto the sample; the emission was dispersed in a single-grating spectrometer integrated into the Flurolog and detected with a nitrogen-cooled InGaAs diode array. UV-vis measurements were performed with an Evolution Array Spectrophotometer from Thermo-Fisher, wavelength range 270-1100 nm, and IR absorption measurements in the 950-1320 nm range were performed with a Perkin-Elmer Lambda 950.

\section{AuNR SYNTHESIS AND CHARACTERIZATION.}

The AuNR were synthesized according to a slight modification of the seed mediated growth developed by Jana et al. [S-1] and Nikoobakht et al. [S-2]. All the chemicals listed here were obtained from Sigma Aldrich. The gold seeds were prepared via the following method: A $5 \mathrm{ml}$ distilled water solution containing $0.125 \mathrm{mM}$ $\mathrm{HAuCl}_{4}$ (tetrachloroauric acid trihydrate) and $0.1 \mathrm{M} \mathrm{CTAB} \mathrm{(Hexadecyltrimethylammoniumbromid)} \mathrm{was}$ warmed to $30^{\circ} \mathrm{C}$ in order to dissolve the CTAB. While strongly stirring, we added to the solution a volume of $0.6 \mathrm{~mL}$ of freshly made $0.01 \mathrm{M} \mathrm{NaBH}_{4}$ (sodium borohydride), that caused the sudden formation of the gold nanoseeds and made the solution turn light brown. The resulting gold nanoseeds had a diameter of around $1.5 \mathrm{~nm}$ and were reactive for 2-6 hours.

In order to grow the nanostructures out of the nanoseeds, we prepared $10 \mathrm{~mL}$ of a growth solution containing $0.1 \mathrm{M} \mathrm{CTAB}$ and $0.5 \mathrm{mM} \mathrm{HAuCl}_{4}$ and added $75 \mu \mathrm{L}$ of ascorbic acid $0.1 \mathrm{M}$. The last step consisted in the addition of $5 \mathrm{mM} \mathrm{AgNO}$ (silver nitrate) and the gold seeds. The fine tuning of the aspect ratio of the AuNR is sensitive to the $\mathrm{CTAB}, \mathrm{AgNO}_{3}$ and gold seed ratio. First of all the silver nitrate is important for the symmetry breaking. In absence of silver, no rods will be formed. Further, the amount of CTAB, thereby the quantity of bromide counter ions, is crucial for the rod formation [S-3]. Having a fixed CTAB concentration, as in our case, the amount of $\mathrm{AgNO}_{3}$ determines mainly the aspect ratio of the formed gold particles. Low to no silver content

Address correspondence to Antonio Setaro, setaro@physik.fu-berlin.de. 
results in spherical gold particles, whereas adding higher amounts of silver than gold seeds results in formation of gold nanorods with higher aspect ratio. After addition of the seeds, the growth solution turned red within a few minutes. The final solutions contained rod-shaped particles and, depending upon the aspect ratio, its color ranges from red to blue or purple. For the AuNRs used in this work, we added $80 \mu \mathrm{L}$ of $\mathrm{AgNO}_{3}$ and $60 \mu \mathrm{L}$ of gold nanoseeds.

In Ref. [22] of the manuscript, it was shown that 3 days of stirring SWNTs in CTAB lead to solubilization and isolation of SWNTs. In order to promote the $\pi$-hybrid formation, we followed a gentle isolation and solubilization method that avoided altering of the coverage of the AuNRs and prevented their reaggregation as well as precipitation. To the aim of isolating the SWNT and including them into the $\pi$-hybrids, we decided to exploit the CTAB in the AuNR suspension without addition of any other surfactant and to go through the stirring procedure. We therefore added a certain amount of SWNTs to the AuNR suspensions and left them stirring over a week; after that we let the suspensions settle down and collected the supernatant. Alternative preparation techniques have also been tried and compared. Details on the preparation and characterization of the starting materials and our samples can be found in the next section.

In order to avoid reaggregation and precipitation of the suspended particles (AuNRs as well as SWNTs) due to depletion forces and exclusion volume effects arising from the surfactant micelle excess, we waited some days at room temperature for the CTAB to crystallize according to the Krafft phenomenon [S-4]. As a matter of fact, the Krafft temperature for $\mathrm{CTAB}$ is approximately $25^{\circ} \mathrm{C}$ and, as the standard working temperature is below this temperature, the formation of $\mathrm{CTAB}$ crystals is favored towards the micelle formation [S-4]. In this way, by collecting the upper part of the supernatant, we ensured to have in our suspensions only CTAB-coated AuNRs and isolated CTAB molecules without any CTAB micelles. We further waited 3 days for the suspensions to settle down.

\section{OPTIMIZATION OF THE PREPARATION CONDITION}

A point that could be debated regards the opportunity of the sequence of treatments we applied on our samples. It would be arguable that stirring, even if suspending tubes, could be profitably replaced by sonication for yielding "better" samples. We performed other sequences of control experiments to check this point, for example we performed previously an independent sonication of SWNTs in CTAB and then we mixed the two solutions; unfortunately this does not yield to an enhancement of the luminescence. Another attempt has been performed by sonication instead of stirring of the nanotubes in the gold solution, and this also has been shown to produce isolated tubes - but unfortunately did not show any enhancement (and also an overall lower emission yield than an equivalent sample without any AuNRs). Another attempt has been performed by solubilizing and isolating SWNTs with surfactant of opposite ionicity with respect to CTAB (for example sodium Dodecylsulfonate (SDS), sodium dodecylbenzosulfonate (SBDS), sodium cholate (SC), polystyrene sulfonate (PSS) among the others) in order to make them bind with CTAB and immobilize electrostatically (following a procedure already exploited in some works in literature [S-5]) the tubes on the AuNRs, but, again, no significant PL enhancement could be observed.

Merging this results with the fact that sonication is worsening the optimized NT@Au system and makes it converting towards a system resembling SWNTs sonicated in CTAB, suggests that sonication is a technique to be carefully applied in this context.

These experiments gave us the feeling that the low-energetic, non-perturbing stirring approach is the best way of optimizing the SWNT-AuNR binding for PL enhancement purposes. Even if it is a gentle technique, it leads 
to SWNT-AuNR complexes bound to a certain extent. As a matter of fact, by centrifuging a stirred sample (prepared following the NT@Au procedure), one ends up with a very weakly emitting SWNTs solution, hinting loss SWNTs beyond the expected loss of AuNRs. By centrifuging a sonicated solution, on the contrary, the NT luminescence remains unaltered while a big amount of AuNRs is taken out. This seems to confirm the idea of an AuNR-SWNT hybrid structure formation.

\section{ADDITIONAL PICTURES}
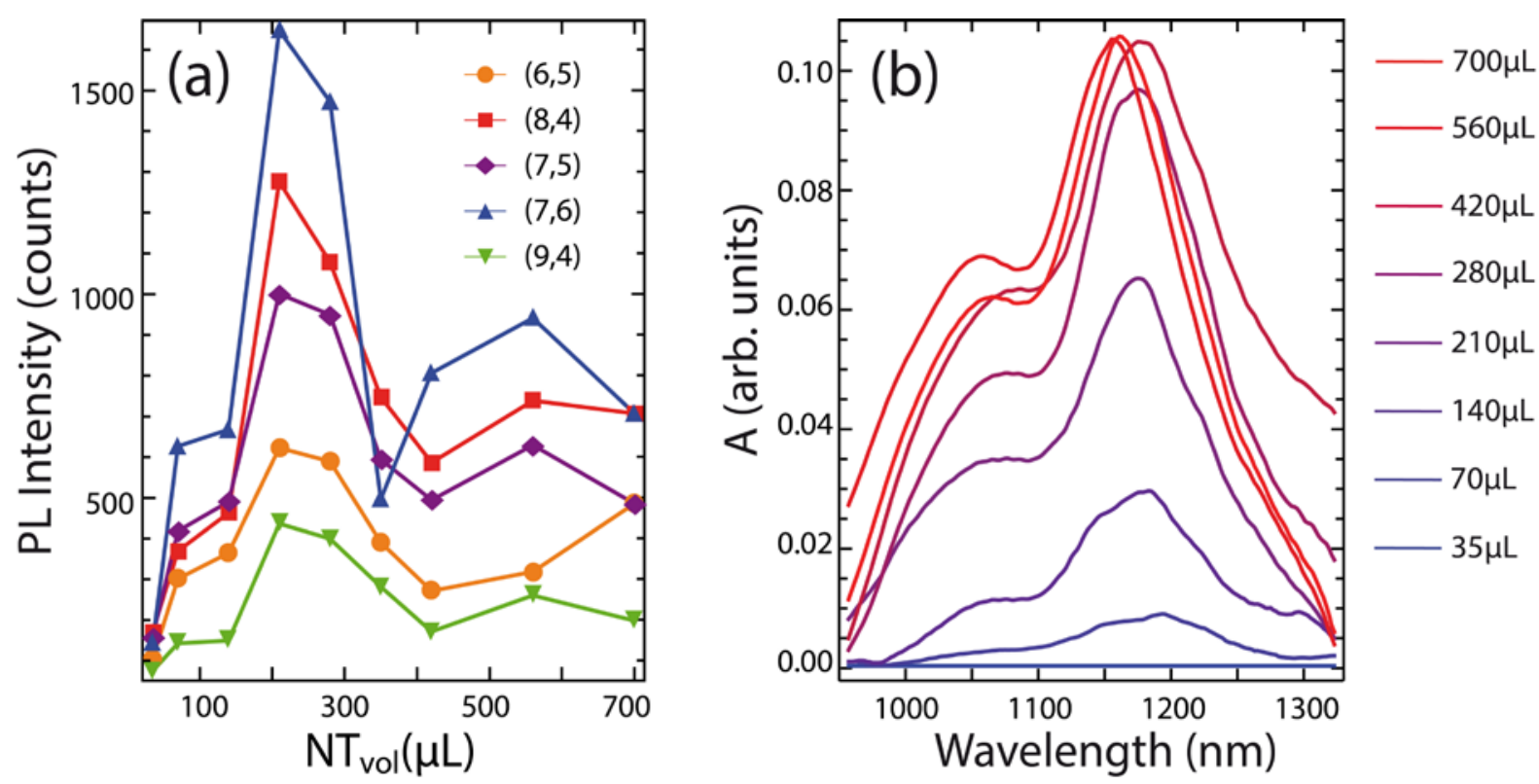

Figure S1 (a) PL emission of tubes with different chiralities for different amount of SWNTs added to the mother solution. (b) IR absorption spectra of the NT@Au suspensions for different amount of SWNTs added to the mother solution.

\section{REFERENCE}

[S-1] Jana, N. R.; Gearheart, L.; Murphy, C . J. Chem. Commun. 2001, 7, 617.

[S-2] Nikoobakht, B.; El-Sayed, M. A. Preparation and Growth Mechanism of Gold Nanorods (NRs) Using Seed-Mediated Growth Method, Chem. Mater. 2003, 15, 1957.

[S-3] Si, S.; Leduc, C.; Delville, M.H.; Lounis, B., Short Gold Nanorod Growth Revisited: The Critical Role of the Bromide Counterion. ChemPhysChem, 2012, 13, 193-202.

[S-4] Dölle, S.; Lechner, B.D.; Park, J.H.; Schymura, S.; Lagerwall, J.P.F.; Scalia, G. Utilizing the Krafft Phenomenon to Generate Ideal Micelle-Free Surfactant-Stabilized Nanoparticle Suspensions, Ang. Chemie Int. Ed. 2012, 51, 3254.

[S-5] Correa-Duarte, M.A.; Sobal, N.; Liz-Marzán, L.M.; Giersig, M., Linear Assemblies of Silica-Coated Gold Nanoparticles Using Carbon Nanotubes as Templates. Adv. Mater., 2004, 16, 2179-2184. 